\title{
Analytical Computation of Information Rate for MIMO Channels
}

\author{
Jinbao Zhang, Zhenhui Tan, and Song Chen \\ Beijing Jiaotong University, Beijing, China \\ Correspondence should be addressed to Jinbao Zhang; jbzhang@bjtu.edu.cn
}

Received 5 September 2016; Revised 10 December 2016; Accepted 4 January 2017; Published 8 February 2017

Academic Editor: Peng Cheng

Copyright (C) 2017 Jinbao Zhang et al. This is an open access article distributed under the Creative Commons Attribution License, which permits unrestricted use, distribution, and reproduction in any medium, provided the original work is properly cited.

Information rate for discrete signaling constellations is significant. However, the computational complexity makes information rate rather difficult to analyze for arbitrary fading multiple-input multiple-output (MIMO) channels. An analytical method is proposed to compute information rate, which is characterized by considerable accuracy, reasonable complexity, and concise representation. These features will improve accuracy for performance analysis with criterion of information rate.

\section{Introduction}

Information rate plays an important role in performance analysis for discrete signaling constellations ( $m$-PSK, $m$ QAM, etc.) [1-4]. Currently, there have been three metrics to evaluate information rate. They are accurate values, lower or upper bounds, and intermediate variables.

According to definition of information rate, direct computation is rather hard for arbitrary fading MIMO channels $[5,6]$. Therefore, Monte Carlo (MC) trials turn out to be a direct and accurate computation [5]. To reduce computational complexity, an improved particle method is proposed in [6]. However, it is iterative and implicit, which makes it ambiguous to analyze. Recently a bitwise computation with concise analytical expression is proposed [4]. Unfortunately, further studies have shown that it is limited to some scenarios, single-input single-output (SISO) and $2 \times 2$ MIMO channels with constellation of BPSK, QPSK, 16QAM, and 64QAM. On the other hand, to the issue of complexity, lower or upper bounds are used to profile information rate instead [7-9]. However, differences between bounds and accurate information rate are still notable. Meanwhile there are also researches which suggest intermediate variables to implement qualitative analysis [10-13]. However, they are handling some special MIMO channels, such as diagonal MIMO channel.

In this work, we are focusing on analytical computation of information rate for arbitrary fading MIMO channels and proposing a symbol-wise algorithm. It is characterized by considerable accuracy, reasonable complexity, and analytical expression, which enable IR to be applicable for analysis. This work is organized as follows. In Section 2, a basic review on analytical computation is presented. Then demonstration of the proposed symbol-wise computation is detailed in Section 3. In Section 4, comparison on accuracy and computational complexity between MC simulation and symbol-wise analytical computation is presented. Finally, conclusions are drawn in Section 5.

\section{Basic Review of Analytical Computation}

Consider the problem of computing information rate

$$
I(\mathbf{s} ; \mathbf{y})=I\left(\left[s_{1}, s_{2}, \ldots, s_{N_{T}}\right]^{T} ;\left[y_{1}, y_{2}, \ldots, y_{N_{R}}\right]^{T}\right)
$$

between input vector $\mathbf{s}=\left[s_{1}, s_{2}, \ldots, s_{N_{T}}\right]^{T}$ and output vector $\mathbf{y}=\left[y_{1}, y_{2}, \ldots, y_{N_{R}}\right]^{T}$ over MIMO channels with additive white Gaussian noise (AWGN). Use $N_{R} \times N_{T}$ dimensional matrix $-\mathbf{H}$ to denote coefficient for arbitrary fading MIMO channels. $N_{T}$ and $N_{R}$ are numbers of transmitting and receiving antennas, respectively. Then we have $[10,13]$

$$
\mathbf{y}=\mathbf{H s}+\mathbf{w}
$$


where $\mathbf{w}$ is AWGN vector. Using Complex-C. $\mathcal{N}\left(0, \sigma_{w}{ }^{2}\right)$ denotes complex Gaussian with zero mean and variance of $\sigma_{w}{ }^{2}$ and $\mathbf{w}$ submits to

$$
\mathbf{w}=\left\{w_{1}, w_{2}, \ldots, w_{N_{R}}\right\}
$$

$w_{k}$ iid. $C \mathcal{N}\left(0, \sigma_{w}{ }^{2}\right), \quad k=1,2, \ldots, N_{R}$.

Every element $s_{k}\left(k=1,2, \ldots, N_{T}\right)$ in $\mathbf{s}$ is selected from the $k$ th finite subconstellation $\boldsymbol{\Omega}_{k}$ uniformly and independently. Therefore, $\mathbf{s}$ is uniformly distributed over finite discrete signaling constellations $-\boldsymbol{\Omega}$, and $\boldsymbol{\Omega}$ is the Cartesian product of all subconstellations. Assuming that size of $\boldsymbol{\Omega}_{k}$ is $N_{k}$, probability density function (PDF) for $\mathbf{s}$ is

$$
\begin{aligned}
p(\mathbf{s}) & =\frac{1}{N} \sum_{k=1}^{N} \delta\left(\mathbf{s}-\mathbf{q}_{k}\right) ; \\
\mathbf{\Omega}=\left\{\mathbf{q}_{1}, \mathbf{q}_{2}, \ldots, \mathbf{q}_{N}\right\}, & \\
N & =\prod_{k=1}^{N_{T}} N_{k} .
\end{aligned}
$$

Provided channel states $\mathbf{-} \mathbf{H}$, definition of information rate gives in [10] as

$$
\begin{aligned}
& I(\mathbf{s} ; \mathbf{y})=\frac{1}{N} \sum_{k=1}^{N} \oint_{\mathbf{W}} \frac{1}{\pi^{N_{R}} \sigma_{w}{ }^{2 N_{R}}} e^{-\|\mathbf{w}\|_{2}{ }^{2} / \sigma_{w}{ }^{2}} \\
& \cdot \log _{2} \frac{e^{-\|\mathbf{w}\|_{2}{ }^{2} / \sigma_{w}{ }^{2}}}{(1 / N) \sum_{m=1}^{N} e^{-\left\|\mathbf{H}\left(\mathbf{q}_{k}-\mathbf{q}_{m}\right)+\mathbf{w}\right\|_{2}{ }^{2} / \sigma_{w}{ }^{2}}} d \mathbf{w},
\end{aligned}
$$

where $\mathbf{W}$ is domain of AWGN vector $-\mathbf{w}$ and $\|\mathbf{a}\|_{2}$ represents 2-norm for vector a. Generally speaking, (5) requires at least $2 N_{R}$ dimensional integral. Therefore, it is difficult to implement directly. And then MC method is used to compute accurate information rate in [8] as

$$
\begin{aligned}
& I(\mathbf{s} ; \mathbf{y})=\lim _{N_{w} \rightarrow \infty}\left\{\frac{1}{N N_{w}}\right. \\
& \left.\quad \cdot \sum_{n=1}^{N_{w}} \sum_{k=1}^{N} \log _{2} \frac{e^{-\left\|\mathbf{w}_{n}\right\|_{2}{ }^{2} / \sigma_{w}{ }^{2}}}{(1 / N) \sum_{m=1}^{N} e^{-\left\|\mathbf{H}\left(\mathbf{q}_{k}-\mathbf{q}_{m}\right)+\mathbf{w}_{n}\right\|_{2}{ }^{2} / \sigma_{w}{ }^{2}}}\right\} .
\end{aligned}
$$

Neither MC computation is simple, nor it can reveal explicit relation between information rate and channel states. Consequently, bitwise computation is developed, using sum of several adjusted Gaussians to approximate PDF of logwise likelihood ratio (LLR), and then information rate is computed by

$$
\begin{aligned}
I(\mathbf{s} ; \mathbf{y}) & \approx \frac{1}{N} \sum_{l=1}^{\log _{2} N} \sum_{k=1}^{N} \int_{-\infty}^{+\infty} p_{l}\left(\ell \mid \mathbf{q}_{k}\right) I_{l}\left(\ell \mid \mathbf{q}_{k}\right) d \ell \\
& \approx \frac{1}{L} \sum_{l=1}^{L} J\left(\sqrt{2} \beta_{l} \sigma_{l}\right),
\end{aligned}
$$

where $L$ means the number of adjusted Gaussians which is determined by preliminary simulations. Adjustment of $\beta_{l}$ is also determined by simulations. $\sigma_{l}^{2}$ denotes variance of the $l$ th Gaussian, defined in [4]. And $J(x)$ is

$$
\begin{aligned}
J(x) & =1-\int_{-\infty}^{+\infty} \frac{1}{\sqrt{2 \pi} x} e^{-\left(\ell-x^{2} / 2\right)^{2} / 2 x^{2}} \log _{2}\left(1+e^{-\ell}\right) d \ell \\
& = \begin{cases}a_{1} x^{3}+b_{1} x^{2}+c_{1} x: & x \leq 1.6363 \\
1-e^{a_{2} x^{3}+b_{2} x^{2}+c_{2} x+d_{2}}: & x>1.6363 .\end{cases}
\end{aligned}
$$

This bitwise computation achieves acceptable accuracy for single-input single-output (SISO) and $2 \times 2$ MIMO channels with BPSK, QPSK, 16QAM, and 64QAM [4].

\section{Proposed Analytical Computation}

Since that bitwise computation of information rate is limited to some scenarios, we propose a symbol-wise algorithm. In this section, we present strict demonstration and extend this computation to general MIMO scenarios with the help of mutual distance vector as

$$
\mathbf{d}_{k, m}=\mathbf{q}_{k}-\mathbf{q}_{m}
$$

Information rate (5) is rewritten as

$$
\begin{aligned}
& I(\mathbf{s} ; \mathbf{y})=-\frac{1}{N} \sum_{k=1}^{N} \int_{-\infty}^{+\infty} p_{w_{k, m}^{\prime}}(w) \\
& \cdot \log _{2}\left(\frac{1}{N} \sum_{m=1}^{N} e^{-\left\|\mathbf{H d}_{k, m}\right\|_{2}{ }^{2}+w_{k, m}^{\prime} / \sigma_{w}{ }^{2}}\right) d w
\end{aligned}
$$

where

$$
w_{k, m}^{\prime}=\operatorname{tr}\left(\mathbf{H d}_{k, m} \mathbf{w}^{H}+\mathbf{w} \mathbf{d}_{k, m}{ }^{H} \mathbf{H}^{H}\right) .
$$

Because $\mathbf{w}$ is AWGN vector, the PDF of $w_{k, m}^{\prime}$ is Gaussian,

$$
w_{k, m}^{\prime} \sim \mathcal{N}\left(0,2\left\|\mathbf{H d}_{k, m}\right\|_{2}^{2} \sigma_{w}^{2}\right)
$$

And $\mathcal{N}\left(0,2\left\|\mathbf{d}_{k, m}\right\|_{2}{ }^{2} \sigma_{w}{ }^{2}\right)$ denotes Gaussian with zero mean and variance of $2\left\|\mathbf{d}_{k, m}\right\|_{2}{ }^{2} \sigma_{w}{ }^{2}$. Normalize Gaussian variance as

$$
\begin{aligned}
& w \sim \mathcal{N}(0,1) ; \\
& w=\frac{w_{k, m}^{\prime}}{\sqrt{2}\left\|\mathbf{H d}_{k, m}\right\|_{2} \sigma_{w}} .
\end{aligned}
$$

We have

$$
\begin{aligned}
& I(\mathbf{s} ; \mathbf{y})=-\frac{1}{N} \sum_{k=1}^{N} \int_{-\infty}^{+\infty} \frac{1}{\sqrt{2 \pi}} e^{-w^{2} / 2} \\
& \cdot \log _{2}\left(\frac{1}{N} \sum_{m=1}^{N} e^{-\left\|\mathbf{H d}_{k, m}\right\|_{2}{ }^{2} / \sigma_{w}{ }^{2}-\sqrt{2}\left\|\mathbf{H d}_{k, m}\right\|_{2} / \sigma_{w}{ }^{w}}\right) d w .
\end{aligned}
$$


Consequently, it is pivotal to compute numerical integral. Use Taylor expansion as

$$
\begin{gathered}
\int_{-\infty}^{+\infty} \frac{1}{\sqrt{2 \pi}} e^{-w^{2} / 2} \log _{2}\left(\frac{1}{N} \sum_{m=1}^{N} e^{-a_{m}{ }^{2}-\sqrt{2} a_{m} w}\right) d w \\
=\int_{-\infty}^{+\infty} \frac{1}{\sqrt{2 \pi}} e^{-w^{2} / 2} \\
\cdot \log _{2}\left[\sum_{p=0}^{+\infty} \sum_{n=0}^{+\infty} \frac{(-1)^{p+n} 2^{p / 2}}{p ! n !} \chi_{p+2 n} w^{p}\right] d w .
\end{gathered}
$$

The denotation $\chi_{p+2 n}$ is defined as

$$
\chi_{p+2 n}=\sum_{m=1}^{N} \frac{a_{m}^{p+2 n}}{N} .
$$

Equation (16) points out that $\chi_{p+2 n}$ is arithmetic mean of progression $\left[a_{1}, a_{2}, \ldots, a_{N}\right]$ to the power of $(p+2 n)$. For the sake that it is difficult to compute (15) with $\chi_{p+2 n}$ directly, a suboptimal computation is proposed. Regarding $\left\{\chi_{p+2 n}\right\}$ as a progression of $N$ elements, we are trying to find another geometric progression $\left\{\chi^{p+2 n}\right\}$. This geometric progression $\left\{\chi^{p+2 n}\right\}$ is characterized by minimum mean square error to the original progression $\left\{\chi_{p+2 n}\right\}$. And then, this characteristic guarantees the minimum mean square error between computations of (15) with $\left\{\chi^{p+2 n}\right\}$ and $\left\{\chi_{p+2 n}\right\}$. Consequently, we accomplish this geometric progression with least-squares fitting,

$$
\chi=\min _{\chi}\left\{\lim _{R \rightarrow+\infty}\left[\frac{1}{R} \sum_{r=1}^{R}\left(\chi^{r}-\chi_{r}\right)^{2}\right]\right\} .
$$

Numerical approximation gives

$$
\chi^{2} \approx-\log _{e}\left(\frac{1}{N} \sum_{m=1}^{N} e^{-\chi_{m}^{2} /\left(3-e^{-\chi_{m}^{2} / 4}\right)}\right) .
$$

Thus, integral is approximated as

$$
\begin{aligned}
& \int_{-\infty}^{+\infty} \frac{1}{\sqrt{2 \pi}} e^{-w^{2} / 2} \log _{2}\left(\frac{1}{N} \sum_{m=1}^{N} e^{-a_{m}{ }^{2}-\sqrt{2} a_{m} w}\right) d w \\
& \approx \int_{-\infty}^{+\infty} \frac{1}{\sqrt{2 \pi}} e^{-w^{2} / 2} \\
& \cdot \log _{2} \sum_{p=0}^{+\infty} \sum_{n=0}^{+\infty}\left(\frac{(-1)^{p+n} 2^{p / 2} w^{p} \chi^{p+2 n}}{p ! n !}\right) d w \\
& =\int_{-\infty}^{+\infty} \frac{1}{\sqrt{2 \pi}} e^{-w^{2} / 2} \log _{2}\left(e^{-\chi^{2}-\sqrt{2} \chi w}\right) d w \\
& =\log _{2}\left(\frac{1}{N} \sum_{m=1}^{N} e^{-\chi_{m}^{2} /\left(3-e^{-\chi_{m} / / 4}\right)}\right) \text {. }
\end{aligned}
$$

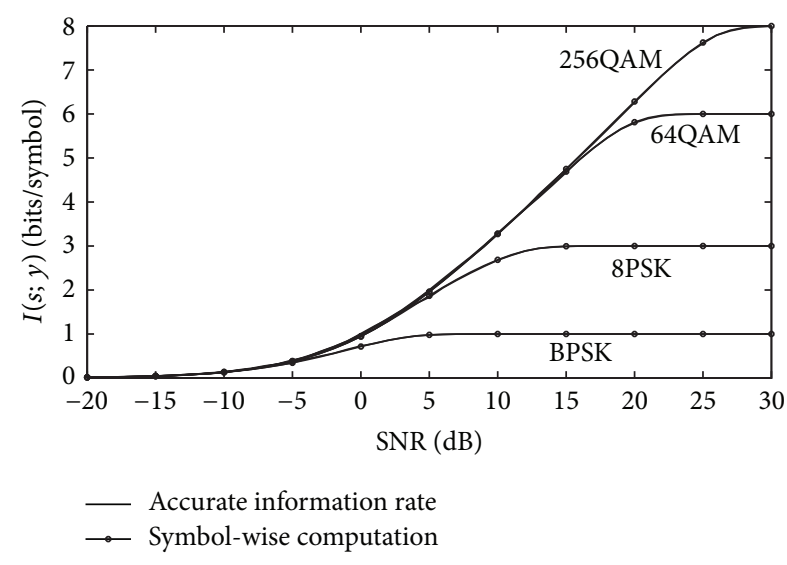

FIGURE 1: Numerical results for SIMO.

Recall (14), we get analytical computation of information rate as

$$
\begin{aligned}
& I(\mathbf{s} ; \mathbf{y}) \approx-\frac{1}{N} \sum_{k=1}^{N} \log _{2}\left(\frac{1}{N}\right. \\
& \left.\cdot \sum_{m=1}^{N} e^{-\left(\left\|\mathbf{H d}_{k, m}\right\|_{2}{ }^{2} / \sigma_{w}{ }^{2}\right) /\left(3-e^{-\left\|\mathbf{H d} d_{k, m}\right\|_{2}{ }^{2} / \sigma_{w} / 4}\right)}\right) .
\end{aligned}
$$

\section{Numerical Results}

This section presents numerical results for validation. Accurate information rate is computed with MC method (6) as basic reference for comparison. It is clear that information rate is determined by digital signaling constellation $\Omega$, channel states $\mathbf{H}$, and AWGN variance $\sigma_{w}{ }^{2}$. To assure that the numerical results are self-contained, we will classify $[\Omega, \mathbf{H}$ and ${\sigma_{w}}^{2}$ ] into several orthogonal spaces.

4.1. Numerical Results for Arbitrary Fading SIMO Channels. We analyze single-input multiple-output (SIMO) channels first. The simplest scenario, single-input single-output (SISO) channels, can be seen as a subset of SIMO. Consider

$$
\mathbf{y}=\mathbf{H} s+\mathbf{w}
$$

with maximum ration combination (MRC); this transmission is effective to

$$
\begin{aligned}
y^{\prime} & =s+w^{\prime} \\
w^{\prime} & \sim \mathcal{N}\left(0, \frac{\sigma_{w}{ }^{2}}{\|\mathbf{H}\|_{2}{ }^{2}}\right) .
\end{aligned}
$$

For generality, constellations BPSK, 8PSK, 64QAM, and 256QAM are assigned to $s$, respectively. Numerical results on computation of information rate are illustrated in Figure 1. It is clear that the proposed method achieves considerable accuracy and tells the accurate information rate for different digital signaling constellations. 


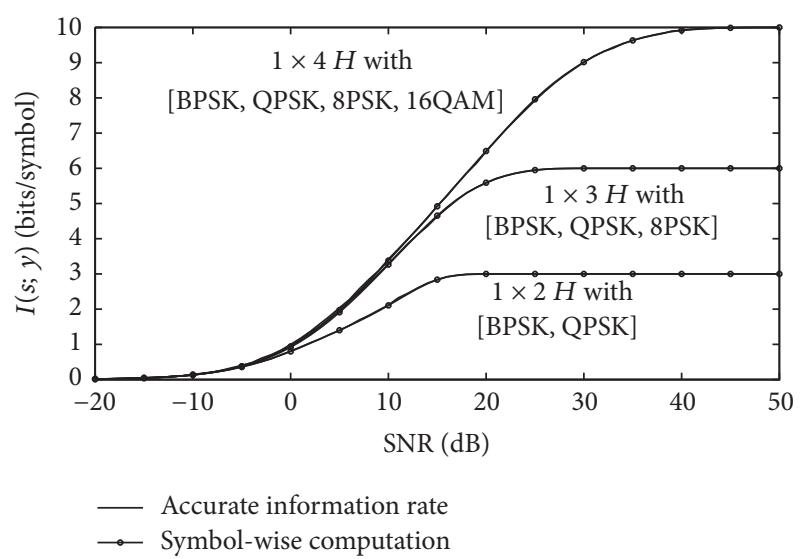

Figure 2: Numerical results for MISO.

4.2. Numerical Results for Arbitrary Fading MISO Channels. Then we consider multiple-input single-output (MISO) channels. Consider example as follows:

$$
y=\left[h_{1}, h_{2}, \ldots, h_{N_{T}}\right]\left[s_{1}, s_{2}, \ldots, s_{N_{T}}\right]^{T}+w .
$$

Since $h_{k}\left(k=1,2, \ldots, N_{T}\right)$ is complex, MISO channel is of at least $2\left(N_{T}-1\right)$ degrees freedom, so it is impossible to profile full classification. Therefore, we have to make the following yields.

(1) $N_{T}$ is selected as 2, 3, and 4, for example.

(2) For each value of $N_{T}, h_{k}\left(k=1,2, \ldots, N_{T}\right)$ is randomly chosen with complex Gaussian.

(3) Assuring generality, constellations BPSK, QPSK, 8PSK, and 16QAM are assigned to each symbol in vector $\mathbf{s}$, respectively.

Numerical results are illustrated in Figure 2. It is also clear that symbol-wise computation achieves considerable accuracy for SIMO channels.

4.3. Numerical Results for Arbitrary Fading MIMO Channels. As to MIMO channel, $\mathbf{H}$ is consisted of $N_{R} \times N_{T}$ complex coefficients, so we make similar yields.

(1) $N_{R}$ is 3 , and $N_{T}$ is selected as 2,3 , and 4 , for example.

(2) All elements of $\mathbf{H}$ are randomly chosen with complex Gaussian.

(3) Assuring generality, constellations BPSK, QPSK, 8PSK, and 16QAM are assigned to each symbol in vector $\mathbf{s}$, respectively.

Numerical results illustrated in Figure 3 show that symbolwise computation achieves considerable accuracy also.

4.4. Numerical Results for Resolution of Information Rate. Consider another problem as computing information rate of any component within input vector accurately. Firstly, it is proven that information rate can be resolved as follows [11]:

$$
I\left(\mathbf{s}_{s} ; \mathbf{y}\right)=I(\mathbf{s} ; \mathbf{y})-I\left(\mathbf{s}_{r} ; \mathbf{y}\right),
$$

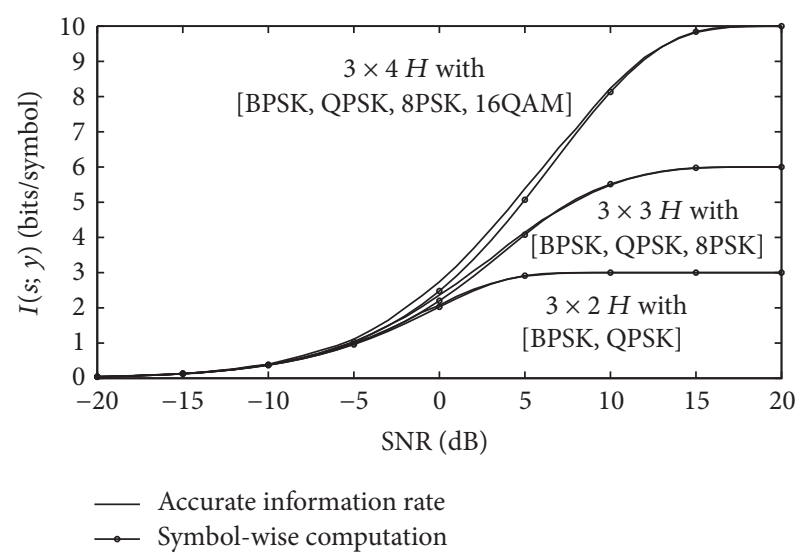

FIgURE 3: Numerical results for MIMO.

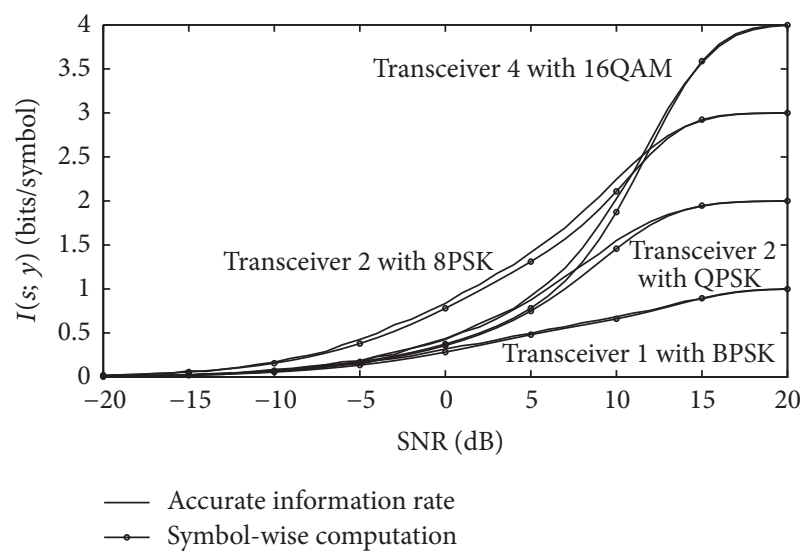

FIgURE 4: Numerical results for resolution of information rate.

where $\mathbf{s}_{r}$ is residual subvector by excluding $\mathbf{s}_{s}$ from $\mathbf{s}$. This tells that we can compute arbitrary information rate provided computation of (5). Consequently, this part of results validates (24) with symbol-wise computation of information rate. For example, that $\mathbf{H}$ is $3 \times 4$ and randomly chosen similarly as previous sections. Used constellations are BPSK, QPSK, 8PSK, and 16QAM for each symbol in vector s, respectively. Interesting components are individual symbol in vector $\mathbf{s}$. Information rate of every transceiver is illustrated in Figure 4. It is the same as before that symbol-wise computation achieves considerable accuracy.

4.5. Numerical Results for Erasure Channel. Besides MIMO channels mentioned before, there is a very special kind of channel as follows:

$$
y=[1,1]\left[s_{1}, s_{2}\right]^{T}+w,
$$

where $s_{1}$ and $s_{2}$ are both BPSK modulated. This kind of transmission is a typical erasure channel, where $N_{R}$ is 1 and $N_{T}$ is 2. Numerical results are illustrated in Figure 5. It also validates the proposed symbol-wise computation. 


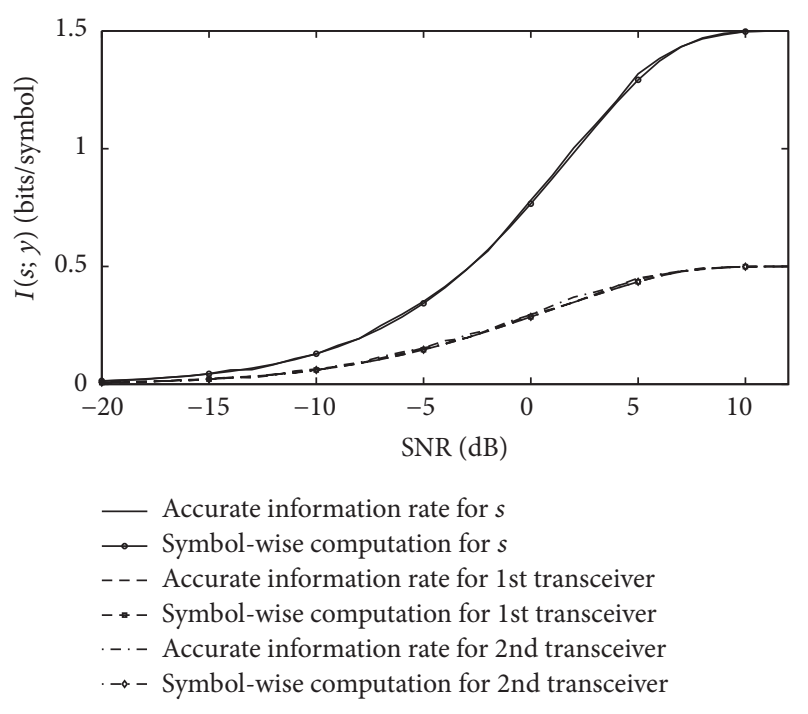

FIGURE 5: Numerical results for erasure channel.

\section{Discussion}

We have presented an analytical computation of information rate for arbitrary fading MIMO channels. Based on simulation, we have further discussion as follows.

For the "Generality" of the proposal, similarly as presented in $[5,6,9,10]$, we demonstrate computation of information rate for MIMO channel, without supplemental conditions except the knowledge of constellations $-\boldsymbol{\Omega}$, power of AWGN, and channel status $\mathbf{-} \mathbf{H}$ to the receiver. And then, we carry out validation with numerical results on selected MIMO cases. To ensure that the selected MIMO cases are general, numbers of transmitting and receiving antenna $\left(N_{T}\right.$, $N_{R}$ ) vary from 1 to 4 , as presented in Section 4; the adopted constellations vary from QPSK to 256QAM; and simulated channel status $\mathbf{-} \mathbf{H}$ are randomly generated. In addition, (24) (in Section 4.4) can be used to compute information rate for each MIMO stream, which also improves generality, whereas bitwise computation is quite limited by tuning factors related to selected MIMO scenarios [4].

As to "Accuracy" of the proposal, validations in Section 4 show that the maximum gap between information rate computed by proposed and MC methods is lower than $0.063 \mathrm{bits} / \mathrm{symbol}$. Reference information rate is computed by MC method [5], and particle method achieves exactly accurate numerical results [6]. With SNR based intermediate variables, estimation [4] and upper/lower bounds $[9,10]$ are proposed. The gap between reference information rate by MC and upper/lower bounds is about 0.15 bits/symbol $[9,10]$, which is a little worse than computation proposed in this work. The accuracy of estimation in [4] is not compared for the sake that there are quite a lot MIMO and constellations cases when information rate is unavailable.

Then we turn to "Complexity." Computation shown as (20) will need $N \times N$ exponential processes and $N$ logarithms, which is approximately equivalent to those presented in [9, 10]. This is much simpler than MC method [5]. The comparison on complexity between the proposed and particle methods is dependent to scale of MIMO and constellation, because it tells in [6] that particle methods need a sequence length of $10^{4}$ to obtain convergent calculation, while the suggested method in this work is of complexity varying with $N$.

To sum up, the proposed computation makes sense that it is interpreted in a general and concise analytical expression, so that it facilitates further studies on performance and optimization of wireless MIMO transmissions with information rate criterion.

\section{Disclosure}

This work was presented in part at 2010 International Conference on Communications.

\section{Competing Interests}

The authors declare that there is no conflict of interests regarding the publication of this paper.

\section{Acknowledgments}

This work is financially supported by National Natural Science Foundation of China (NSFC) 61471030 and 61631013 and Research Project of Railway Corporation (2016J011-H).

\section{References}

[1] J. Hu, T. M. Duman, M. F. Erden, and A. Kavcic, "Achievable information rates for channels with insertions, deletions, and intersymbol interference with i.i.d. inputs," IEEE Transactions on Communications, vol. 58, no. 4, pp. 1102-1111, 2010.

[2] R.-R. Chen and R. Peng, "Performance of channel coded noncoherent systems: modulation choice, information rate, and Markov chain Monte Carlo detection," IEEE Transactions on Communications, vol. 57, no. 10, pp. 2841-2845, 2009.

[3] J. Zhang, H. Zheng, Z. Tan, Y. Chen, and L. Xiong, "Link evaluation for MIMO-OFDM system with ML detection," in Proceedings of the (ICC '10)-2010 IEEE International Conference on Communications, pp. 1-6, Cape Town, South Africa, May 2010.

[4] K. Sayana, J. Zhuang, and K. Stewart, "Short term link performance modeling for ML receivers with mutual information per bit metrics," in Proceedings of the IEEE Global Telecommunications Conference (GLOBECOM '08), pp. 4313-4318, New Orleans, La, USA, December 2008.

[5] A. B. Owen, "Monte Carlo extension of quasi-Monte Carlo," in Proceedings of the 30th Conference on Winter Simulation (WSC '98), vol. 16, pp. 571-577, Washington, DC, USA, December 1998.

[6] J. Dauwels and H.-A. Loeliger, "Computation of information rates by particle methods," IEEE Transactions on Information Theory, vol. 54, no. 1, pp. 406-409, 2008.

[7] N. Guney, H. Deliç, and F. Alagöz, "Achievable information rates of PPM impulse radio for UWB channels and rake reception," IEEE Transactions on Communications, vol. 58, no. 5, pp. 1524$1535,2010$.

[8] A. Steiner and S. Shamai, "Achievable rates with imperfect transmitter side information using a broadcast transmission 
strategy," IEEE Transactions on Wireless Communications, vol. 7, no. 3, pp. 1043-1051, 2008.

[9] P. Sadeghi, P. O. Vontobel, and R. Shams, "Optimization of information rate upper and lower bounds for channels with memory," IEEE Transactions on Information Theory, vol. 55, no. 2, pp. 663-688, 2009.

[10] O. Shental, N. Shental, S. Shamai, I. Kanter, A. J. Weiss, and Y. Weiss, "Discrete-input two-dimensional Gaussian channels with memory: estimation and information rates via graphical models and statistical mechanics," Institute of Electrical and Electronics Engineers. Transactions on Information Theory, vol. 54, no. 4, pp. 1500-1513, 2008.

[11] P. Sadeghi and P. Rapajic, "On information rates of time-varying fading channels modeled as finite-state Markov channels," IEEE Transactions on Communications, vol. 56, no. 8, pp. 1268-1278, 2008.

[12] X. Jin, J.-D. Yang, K.-Y. Song, J.-S. No, and D.-J. Shin, “On the relationship between mutual information and bit error probability for some linear dispersion codes," IEEE Transactions on Wireless Communications, vol. 8, no. 1, pp. 90-94, 2009.

[13] W. Dai, Y. Liu, B. Rider, and V. K. Lau, "On the information rate of MIMO systems with finite rate channel state feedback using beamforming and power on/off strategy," IEEE Transactions on Information Theory, vol. 55, no. 11, pp. 5032-5047, 2009. 


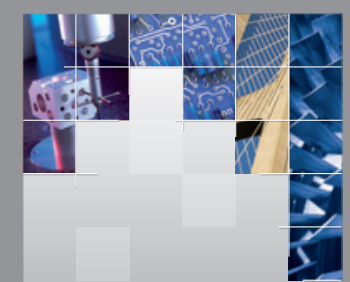

\section{Enfincering}
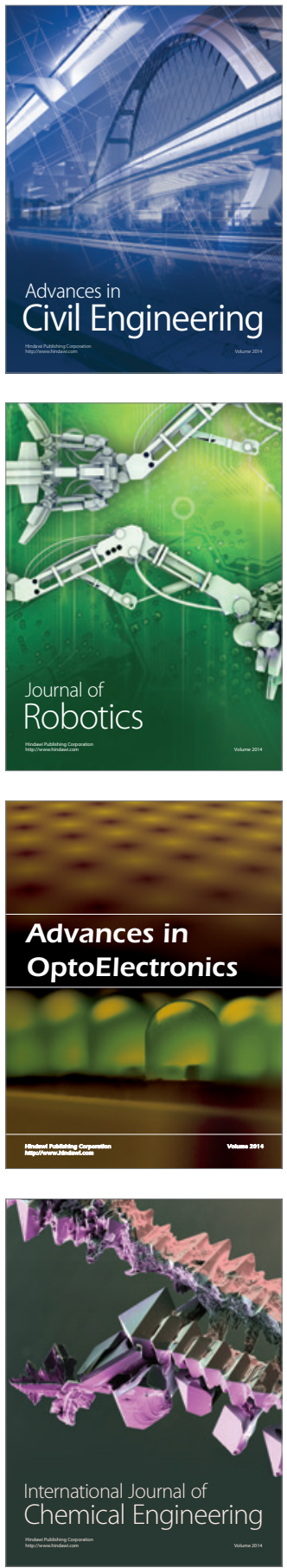

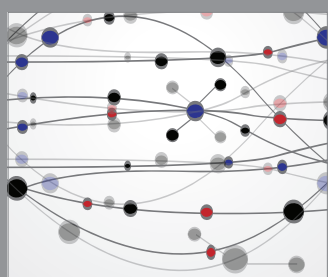

The Scientific World Journal

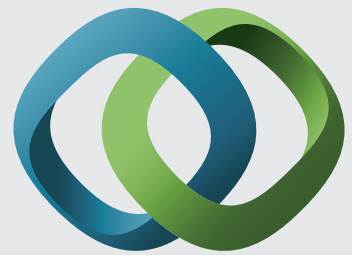

\section{Hindawi}

Submit your manuscripts at

https://www.hindawi.com
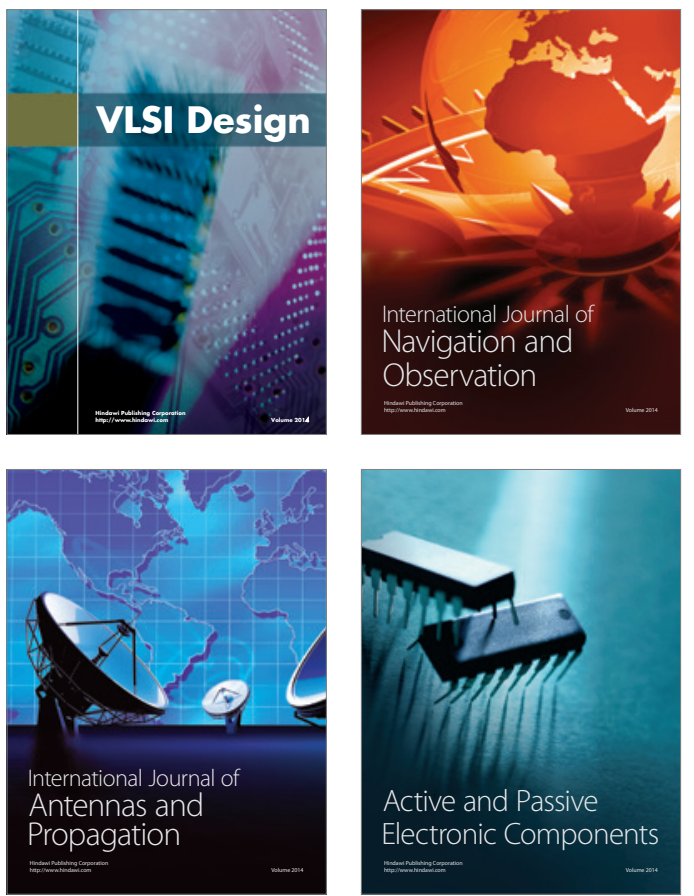
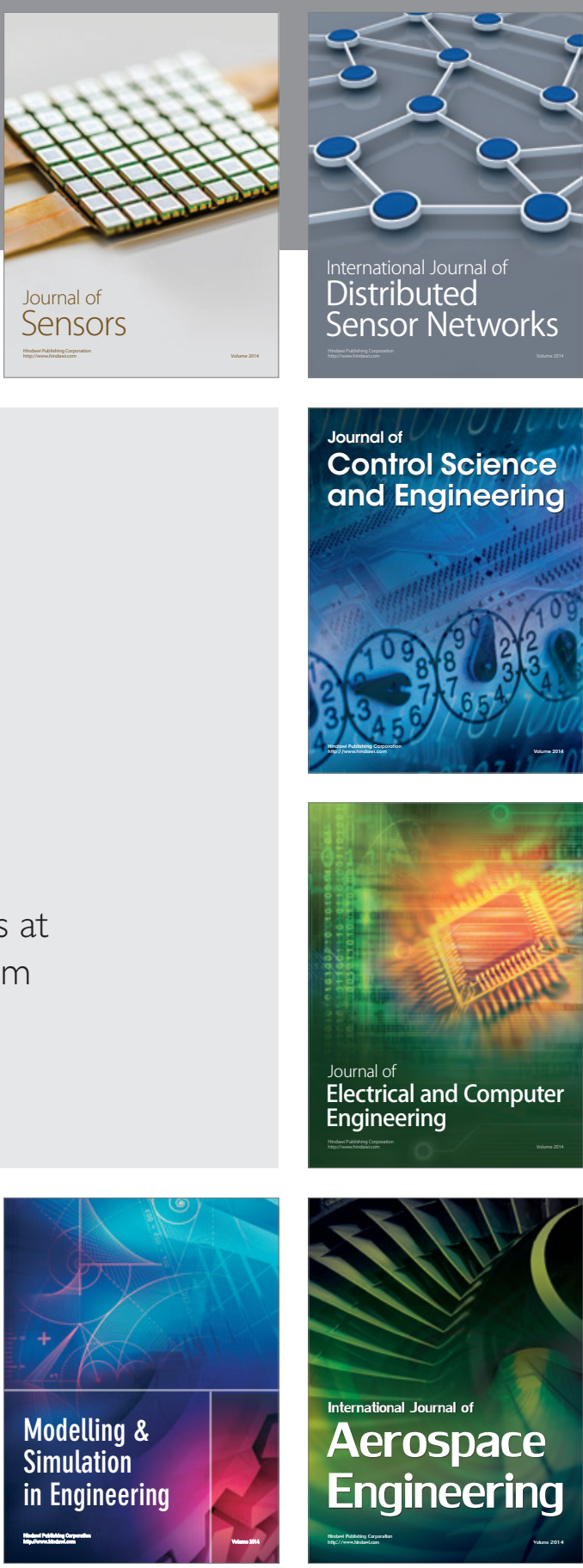

International Journal of

Distributed

Sensor Networks

$-$

Joumal of

Control Science

and Engineering
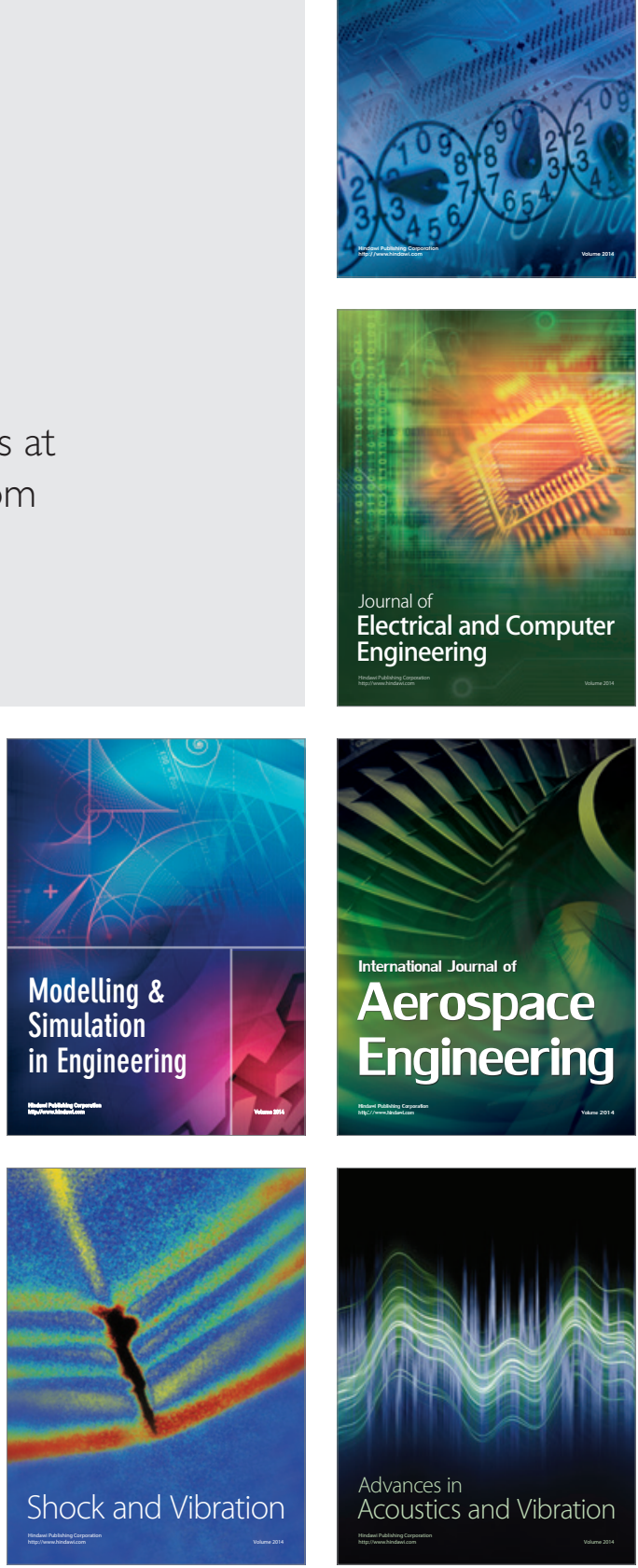